ORIGINAL ARTICLE

\title{
Clinical Pathology and Prognostic Analysis of Visceral Metastatic Carcinoma of Unknown Primary Site (VMCUP)
}

\author{
Q Xu ${ }^{1}$, J Jiang $^{1 *}$, S Yang ${ }^{1,2,3^{*}}$, X Wang ${ }^{1}, \mathrm{~J} \mathrm{Wang}^{1}$, Q Chen ${ }^{1,2,3}$
}

\begin{abstract}
Objective: To analyse the clinicopathologic characteristics of visceral metastatic carcinoma of unknown primary site (VMCUP), discuss the relationship between its treatment and prognosis, and provide the basis for individualized clinical diagnosis and treatment.

Methods: A retrospective analysis on clinical pathologic data was performed on 21 VMCUP cases from Fujian Union Hospital from January 2007 to January 2012. Follow-up visits on patients were made and analysis of their survival conditions and relevant influencing factors were collated.

Results: The gender distribution of the 21 VMCUP cases was 1:1.1; the median age was 63 years and the pathology type was dominated by adenocarcinoma. The median survival time and survival rate in patients accepting the treatment were evidently higher than in those patients not accepting the treatment. The median survival time of those patients receiving more than one treatment procedure was higher than in those patients only receiving chemotherapy. Among those patients who received comprehensive therapy, the median survival time in those mainly receiving operative therapy was also higher than in those only receiving chemotherapy $(\mathrm{p}<0.05)$. The median survival time in those receiving chemotherapy more than two cycles or more than four cycles was evidently longer than those receiving chemotherapy two or less cycles or four or less cycles separately. For those choosing paclitaxel in combination with platinum as first-line chemotherapy, their median survival time was longer than those primarily taking fluorouracil $(\mathrm{p}<0.05)$. In addition, there were no statistical differences ( $\mathrm{p}>0.05)$ in the median survival times among groups receiving the same treatment but with different age, gender or pathological types. It was indicated by multiple-factor analysis that the chemotherapeutics, chemotherapy times and treatment methods were prognostic factors affecting the survival of VMCUP.

Conclusion: The overall progression of VMCUP patients deteriorates quickly, with a poor prognosis and without a standard treatment pattern. Appropriate chemotherapy, surgery and radiotherapy, however, could play an active role in controlling the state of illness. For the patients who accepted chemotherapy, the curative effect could be further improved with the increase of chemotherapy cycles and application of new drugs, such as paclitaxel, and comprehensive treatment could more effectively prolong the survival time of patients.
\end{abstract}

Keywords: Internal organs, prognosis, treatment, visceral metastatic carcinoma of unknown primary site (VMCUP)

WIMJ Open 2014; 1 (3): 117

From: ${ }^{1}$ Teaching and Research Department of Oncology, Union Clinical Medical College of Fujian Medical University, Fuzhou 350001, China, ${ }^{2}$ Department of Internal Medicine Oncology, Fujian Medical University Union Hospital, Fuzhou 350001, China and ${ }^{3}$ Fujian Provincial Key Laboratory of Tumor Translational Medicine, Fuzhou 350001, China.

Correspondence: Dr Q Chen, Department of Internal Medicine Oncology, Fujian Medical University Union Hospital, Fuzhou, Fujian, China 350001. E-mail: drcqiang@126.com

*Co-first authors

\section{INTRODUCTION}

Visceral metastatic carcinoma of unknown primary site (VMCUP) is featured by metastases with unknown primary site. With the rapid developments in technology, if carcinoma of unknown primary site could be detected earlier and treated promptly, the overall survival time could be extended. Visceral metastatic carcinoma of unknown primary site is relatively rare clinically, with its morbidity accounting for only $3 \sim 5 \%$ in 2011 . Its occurrence rate ranks 
in the top 10 among global common malignant tumours, and its mortality ranks fourth $(1-3)$. This paper reports a retrospective analysis of the treatment of 21 VMCUP cases, with complete clinical pathology documentation from Fujian Medical University Union Hospital, over five years from January 2007 to January 2012, and includes a discussion with relevant documented literature.

\section{SUBJECTS AND METHODS \\ Clinical data}

The metastasis of these 21 VMCUP cases was verified through histology or cytology examination. No primary malignant tumour was found after a detailed clinical examination, as well as regular laboratory, tumour marker, isotope, endoscope and video examinations etc. The patients had no medical history of malignant tumour or of an unknown resection. Ten patients were males and 11 were females. The gender ratio was 1:1.1. The ages of the patients ranged from 35-82 years old and the median age was 63 years. Fourteen cases had adenocarcinoma (66.7\%), four had squamous carcinoma (19.0\%), and three had other types. The metastasis in these 21 patients involved liver, lungs, ovary, throat, pleura and peritoneum; 20 cases (95.2\%) had multifoci and one $(4.8 \%)$ had single foci.

\section{Therapeutic methods}

Consent was obtained from the patients or patients' parent/caregiver. Among the 21 cases, six cases (28.6\%) were given no treatment. For the 15 cases receiving treatment, 11 cases $(52.4 \%)$ were given only chemotherapy, one case $(4.8 \%)$ had surgery and chemotherapy, two cases $(9.5 \%)$ had radiotherapy and chemotherapy, and one case $(4.8 \%)$ had surgery, chemotherapy and radiotherapy. For the 15 cases receiving chemotherapy, six $(40.0 \%)$ were given firstline paclitaxel with platinum-based chemotherapy, four cases $(26.7 \%)$ had first-line fluorouracil-based chemotherapy and five cases $(33.3 \%)$ had other chemotherapy agents, such as gemcitabine and pemetrexed disodium. The follow-up study was carried out by telephone and mail, and ranged from 0.4 90 months. The therapeutic evaluation was according to the World Health Organization (WHO) evaluation standard.

\section{Statistical methods}

The survival time was summarized by SPSS (version 20.0). Log-rank test method was used to compare the survival rate. The difference was statistically significant when $p<0.05$. Relative risk (RR) value was used to describe the results (4).

\section{RESULTS}

\section{Survival time and survival rate of VMCUP}

All the 21 VMCUP patients were followed-up. The overall survival time for the 21 patients was $0.4 \sim 90$ months, with the median survival time of 4.4 months. The survival time for the 15 patients receiving treatment was $0.7 \sim 90$ months, with the median survival time of 9.0 months. The one-year and two-year survival rates were $40.0 \%$ and $13.3 \%$, respectively. In contrast, the survival rate for the six patients not accepting treatment was $0.4 \sim 4.2$ months, with the median survival rate of only 1.3 months. And the one-year and two-year survival rates were 0 . For these $21 \mathrm{VMCUP}$ patients, the survival time between those accepting treatment or not had statistically significant difference $\left(\chi^{2}=10.509, p=0.001\right)$; and the one-year and two-year survival rates between these two groups were significantly different as well $\left(\chi^{2}=0.019\right.$, $p=0.001)$.

\section{Relationship between treatment methods and prognosis of VMCUP}

Among the 15 VMCUP patients receiving treatment, 11 patients $(73.3 \%)$ received only chemotherapy and four patients $(26.7 \%)$ received comprehensive therapy. The survival time for the group receiving only chemotherapy was 0.7 17 months, with the median survival time of 5.0 months. The survival time for the group receiving comprehensive treatment was 1.0 90 months, with the median survival time of 39 months. The survival times were significantly different between these two groups $\left(\chi^{2}=5.402, p=0.020\right)$. For the two patients who also underwent surgery in the comprehensive treatment group, their median survival time was 39 months. Compared with the group only receiving chemotherapy, the differences in the survival time were also statistically significant $\left(\chi^{2}=4.644, p=0.031\right)$.

\section{Relationship between chemotherapy cycles and prognosis of VMCUP}

The 11 patients receiving only chemotherapy were analysed by the number of chemotherapy cycles they received. Five patients received two or less chemotherapy cycles, while the other six patients received more than two chemotherapy cycles. Their median survival time was 4.4 months and 9.0 months, respectively. The survival times were significantly different between these two groups $\left(\chi^{2}=4.919, p=0.027\right)$. Of the 15 patients receiving chemotherapy, seven received four or less chemotherapy cycles, while eight patients received more than four chemotherapy cycles. Their median survival time was 4.4 months and 12.1 months, respectively. The survival times of these two groups were significantly different $\left(\chi^{2}=8.675, p=0.003\right)$.

\section{Relationship between selection of first-line chemotherapy drugs and prognosis of VMCUP}

For the 11 patients receiving only chemotherapy, the first-line plan selected was paclitaxel in combination with platinum, fluorouracil, or other drugs. The survival rate of these three groups was 8.1 months, 1.0 month, and 4.4 months, respectively. Comparisons were made between two of them. The survival time between the therapy groups with paclitaxel in combination with platinum or with fluorouracil was significantly different $\left(\chi^{2}=6.000, p=0.014\right)$. 


\section{Relationship between age, gender and prognosis of VMCUP}

Among the 15 VMCUP patients receiving treatment, the ratio of male and female was 1:2, and their survival time was 3.9 months and 4.9 months, respectively. The survival time between gender showed no statistically significant difference $\left(\chi^{2}=0.943, p=0.332\right)$. In addition, among the 15 VMCUP patients receiving treatment, the ratio between those $\leq 60$ years old and those $>60$ years old was 7:8, and their median survival time was 4.9 months and 0.7 month, respectively. The survival time of these two age groups showed no statistically significant difference $\left(\chi^{2}=0.005, p=0.944\right)$.

\section{Relationship between pathologic types and prognosis of VCUP}

Among the 15 VMCUP patients receiving treatment, there were 10 , four and one patient who had adenocarcinoma, squamous carcinoma, and cancers of other types, respectively. The median survival time of the three groups was 1.3 months, 4.9 months, and 35 months, respectively. There was no statistically significant difference in the survival time for these three groups $\left(\chi^{2}=3.365, p=0.186\right)$.

\section{Cox regression multi-factor analysis of prognostic factors influencing the survival}

Cox regression multi-factor analysis was conducted on the 15 VMCUP patients receiving treatment by the means of the first-line chemotherapeutic drugs, chemotherapeutic times, and therapeutic methods etc. The first-line chemotherapeutic drugs adopted and prognosis of survival presented a median relevance (the RR value was 0.490 ). The chemotherapeutic times also showed a median relevance on the prognosis of survival $(R R$ value $=2.554)$. The therapeutic methods had the most important influence on the prognosis of survival, and presented a significant relevance $(R R$ value $=0.148)$.

\section{DISCUSSION}

According to long-term research, all metastatic carcinomas should have their primary sites based on the mechanism of tumour invasion and metastasis. However, for a considerable number of patients, the primary sites of carcinoma still cannot be diagnosed, even with state-of-the-art testing methods. These patients have carcinoma of unknown primary origin [CUP] (5-6). Because CUP lacks specific phenotype and biological characteristics of metastasis and invasion, and is unknown in the early phase, it poses many challenges to doctors, in comparison with common malignancies (7). Therefore, it is still of significant importance to further explore the diagnosis and treatment of CUP.

Currently, researchers have conducted extensive and in-depth studies of CUP, but they conclude with different opinions on its classification. Previously, CUP was classified into four categories: CUP adenocarcinoma, CUP squamous carcinoma, CUP low undifferentiated carcinoma and CUP neuroendocrine carcinoma based on the pathological types
(8). Some researchers also classified it into favourable subsets and unfavourable subsets, based on the reaction and prognosis from CUP toward therapy. Alternatively, the National Institute for Health and Care Excellence (NICE) briefly classified it into two types: metastatic epithelium carcinoma and neuroendocrine neoplasm (9). The term 'viscera' refers to a general category of organs directly or indirectly connected with the outside by a means of pipes inside the body cavity. It generally includes the digestive system, respiratory system, urinary system and genital system, which are mainly located in the chest, enterocoelia and pelvic cavity. Some are located at the head, neck and perineum. In morphogenesis, pleura, peritoneum and perineum are closely related to the viscera, and are also a category of the splanchnologia (10). The viscera play an important role in physiological functions of the human body. However, there is a predilection for tumour metastasis, which has a very high mortality. Lin et al once classified CUP into two types in 2002 based on the organ of metastasis, ie the lymphatic metastasis carcinoma and other viscera metastasis carcinoma (11). In 2006, Lin et al further subdivided CUP into four types: CUP of neck lymphatic metastasis, CUP of osseous metastasis, CUP of skin and soft tissues, and VMCUP (11). The analysis on CUP of neck lymphatic metastasis and CUP of osseous metastasis is common, but the reports on VMCUP are still limited. In this study, when a detailed health check was conducted in the 21 VMCUP patients, image examination in combination with serum tumour marker and endoscopy etc were first selected according to conditions for primary site screening. After the pathological tissue or cytology verification was obtained, they confirmed the diagnosis of VMCUP.

The overall progression of CUP patients deteriorated quickly, and multiple foci were usually discovered. When the biological characteristic of malignancy, invasion and metastasis was presented, CUP patients usually had a poor prognosis. The overall survival time of the 21 VMCUP patients in the study was 0.4 90 months, with the median survival time of 4.4 months. The survival time of six patients not accepting treatment was $0.4 \sim 4.2$ months; the median survival time was only 1.3 months and the one-year or twoyear survival time was 0 . Most CUPs have a short survival time. Most of the up-to-date treatment methods of CUP are empirical, without a unified effective therapy. The major measure of whole body therapy is chemotherapy, with big differences in plan, course, dose and administration route. As for exeresis and radiotherapy in local therapy, there are still controversies in indications of operation, excision extension and degree for the former, and their applications are mostly restricted. There is no standard for radiotherapy opportunity, pattern and scope for the latter, but comprehensive treatment has been widely accepted by the scholars. The treatment of CUP should be adjusted according to specific conditions and based on different carcinoma. Different specific therapeutic methods should be adapted 
accordingly to different parts, pathological types and phases. Therefore, the treatment of VMCUP patients in the study also followed this principle.

We discovered that appropriate chemotherapy, operation, radiotherapy and other therapies could play an active role in controlling the state of illness. The survival time of 15 patients receiving treatment was 0.7 90 months; their median survival time was 9.0 months, and their one-year and two-year survival rates were $40.0 \%$ and $13.3 \%$, respectively. The median survival time and one-year and two-year survival rates were evidently higher than those not accepting the treatment $(p<0.05)$. The median survival time of the VMCUP patients only accepting chemotherapy with more than two cycles and more than four chemotherapy cycles was evidently longer than those only accepting two or less or four or less chemotherapy cycles, which indicated that effective chemotherapy could improve the survival of patients. With the increase of the chemotherapy cycles, most of the curative effect could be further improved, while the median survival time of those selecting paclitaxel in combination with platinum in first-line chemotherapy plan was longer than those mostly selecting fluorouracil $(p<0.05)$. Comparing the other drugs with the aforementioned two, the comparative difference in median survival time showed no statistical significance, which indicates that the clinical application of new generation paclitaxel drugs has an important influence in improving the chemotherapeutic effect of VMCUP. Some other new drugs, such as gemcitabine and pemetrexed also showed certain potential (12).

The median survival time of the VMCUP patients mostly receiving surgery among those accepting comprehensive therapy was higher than those only receiving chemotherapy, which strongly showed that the comprehensive therapy played a dominant role in the treatment of VMCUP. The clinical practices also proved that radiotherapy alone had limited effect on extensive visceral metastatic carcinoma. Although radiotherapy was not widely applied in the patients receiving comprehensive therapy, it still had a non-ignorable role. In addition, the survival time for the patients receiving treatment but with different ages, gender and pathological types was similar to that in most other prognostic research on CUP, and showed no statistically significant difference among groups. For the 15 VMCUP patients receiving treatment, we discovered that after conducting Cox regression multi-factor analysis on the first-line chemotherapeutic drugs, chemotherapeutic times and therapeutic methods, the survival prognosis of those mainly adopting paclitaxel in combination with platinum and those mainly adopting fluorouracil in the first-line therapy was better than those adopting other drugs. The influence from chemotherapeutic times on survival prognosis was also presented in median relevance, $i e$, the patients with more chemotherapeutic times had better prognosis. The therapeutic method had the most important influence on survival prognosis, which tends to be relevant. These results recon- firmed that a comprehensive treatment was better than single treatment, and the comprehensive treatment had active influences on the survival prognosis of the VMCUP patients.

In this report, we followed previously accumulated experiences, employed comprehensive treatment procedures, and fully carried out individualized diagnosis and treatment plan for a group of VMCUP patients (13-15). Most VMCUP patients already have an extensive scope of invasion upon discovery, and curative therapy should be adopted for those with limited lesions; operative therapy has certain palliative value to those with quickly deteriorating symptoms, and with symptoms such as obstruction and compression. Radiotherapy shows evident palliative effect on pain, spinal cord compression, precava obstruction caused by metastasis and brain metastases. In addition, close attention should be paid to see if the primary site could be exposed during therapy and should be double checked prior to proceeding with corresponding treatment.

\section{REFERENCES}

1. Greenlee RT, Murray T, Bolden S, Wingo PA. Cancer statistics, 2000. CA Cancer J Clin 2000; 50: 7-33.

2. Massard C, Loriot Y, Fizazi K. Carcinomas of an unknown primary origin - diagnosis and treatment. Nat Rev Clin Oncol 2011; 8: 701-10.

3. Matsubara N, Mukai H, Nagai S, Itoh K. Review of primary unknown cancer: cases referred to the National Cancer Center Hospital East. Int J Clin Oncol 2010; 15: 578-82.

4. Li LM. Epidemiology. Beijing: People's Health Press; 2008: 71.

5. Danczak Z, Wroblowa M. Neoplasm metastases with unknown primary foci. Nowotwory 1964; 14: 247-51. In Polish

6. Briasoulis E, Pavlidis N. Cancer of unknown primary origin. Oncologist 1997; 2: 142-52.

7. Pavlidis N, Pentheroudakis G. Cancer of unknown primary site: 20 questions to be answered. Ann Oncol 2010; 21 (Suppl 7): vii303vii307. doi: 10.1093/annonc/mdq278.

8. Natoli C, Ramazzotti V, Ramazzotti V, Nappi O, Giacomini P, Palmeri $\mathrm{S}$ et al. Unknown primary tumors. Biochim BioPhys Acta 2011; 1816: 13-24.

9. National Institute for Health and Care Excellence. Metastatic malignant disease of unknown primary origin: diagnosis and management of metastatic malignant disease of unknown primary origin. NICE guidelines [CG104]. London: National Institute for Health and Care Excellence; 2010

10. Bai S. Systematic anatomy. 2. People's Health Press; 2010: 101.

11. Lin B, Deng Y. Preliminary discussions on diagnosis and treatment of metastasis carcinoma of unknown origin. Journal of Practical Carcinoma 2006; 21: 312-3.

12. Hainsworth JD, Spigel DR, Thompson DS, Murphy PB, Lane CM, Waterhouse DM et al. Paclitaxel/carboplatin plus bevacizumab/ erlotinib in the first-line treatment of patients with carcinoma of unknown primary site. Oncologist 2009; 14: 1189-97.

13. Briasoulis E, Pavlidis N. Cancer of unknown primary origin. Oncologist 1997; 2: 142-52.

14. Ghosh SK, Bandyopadhyay D, Barma KD, Basu S, Roy A. Metastatic melanoma from an unknown primary site presenting as skin-colored nodules and multiple visceral involvement. Skinmed 2012; 10: 396-9.

15. Greco FA. Cancer of unknown primary site: improved patient management with molecular and immunohistochemical diagnosis. Am Soc Clin Oncol Educ Book 2013: 175-81. doi: 10.1200/EdBook AM.2013.33.175. 
Received 09 Oct 2013

Accepted 11 Dec 2013

Published 22 Dec 2014

Online: http://www.mona.uwi.edu/wimjopen/article/1609

(C) Xu et al 2014.

This is an open access article made freely available under Creative

Commons Attribution 4.0 International (CC BY 4.0). Users are free to share, copy and adapt this work as long as the copyright holder (author) is appropriately and correctly credited. See http://creativecommons.org/ licences/by/4.0/deed.en_us for more information. 